\title{
Bazı Özel Matrisler ve Kombinasyonel Özdeşlikler
}

\author{
Fatma Sidre OĞLAKKAYA ${ }^{\mathbf{1}}$, Süleyman SOLAK ${ }^{\mathbf{2}}$ \\ ${ }^{1}$ Osmaniye Korkut Ata Üniversitesi, Fen Edebiyat Fakültesi, Matematik Bölümü, \\ OSMANIYE \\ ${ }^{2}$ Necmettin Erbakan Üniversitesi, Eğitim Fakültesi, Matematik ve Fen Bilimleri Eğitimi \\ Bölümü, KONYA \\ * sidreoglakkaya@gmail.com
}

\begin{abstract}
Öz: Bu çalışma, Fibonacci, Pascal, Stirling ve Bell sayıları gibi özel sayı dizilerini tanıtmak, bu sayı dizilerinin elemanları kullanılarak oluşturulan matrisleri tanımlamak ve bu matrisler arasındaki bazı kombinasyonel özdeşlikleri araştırmak için yapılmıştır.
\end{abstract}

Anahtar Kelimeler: Bell matrisi, Fibonacci matrisi, Kombinasyonel özdeşlikler, Pascal matrisi, Stirling matrisleri

\section{Some Special Matrices and Combinatorial Identities}

Abstract: In the present study, the main aim is to introduce specific number sequences, such as Fibonacci, Pascal, Stirling, and Bell numbers, to define matrices created using the elements of these number sequences and to investigate some combinational identities among these matrices.

Keywords: Bell matrix, Fibonacci matrix, Combinatorial identities, Pascal matrix, Stirling matrices

\section{Giriş}

Fibonacci say1s1, Pascal sayıs1, 1. ve 2. tip Stirling say1lar1 ve Bell sayıs kombinasyonel özdeşliklerin inşası ve analizinde çok önemli yer tutmaktadır. Literatürde bu sayılar ve diziler kullanılarak elde edilen pek çok çalışma mevcuttur. Vajda (1987), Fibonacci ve Lucas sayılarına ait temel kavramları, teoremleri ve genel özellikleri ele almış ve bu sayılar arasındaki dönüşüm bağıntılarını incelemiştir. Öte yandan Ayber (2003), çalışmasında gerçel sayı kümesi üzerinde tanımlanmış bir işlemin Fibonacci sayılarına uygulanmasına yer vermiş, Fibonacci sayıları ile ilgili temel kavram ve özellikleri gözden geçirmiştir.
Roger (1977), çalışmasında Pascal üçgenine benzer aritmetik özelliklere sahip olan bir çeşit üçgen dizinin teorisini geliştirmiştir. Çam (2005), 1. ve 2. tip Stirling sayıları üzerine çalışmış, bu iki tip Stirling sayıları arasındaki bağıntıyı incelemiştir. Aigner (1999), Hankel matrislerinin determinantlarından yararlanarak Bell sayılarından oluşan dizinin bir karakterizasyonunu sağlamıştır.

$\mathrm{Bu}$ çalışmaların yanı sıra Fibonacci, Pascal, 1. ve 2. tip Stirling ve Bell sayı dizilerinin elemanları kullanılarak inşa edilen özel matrislerin birbirleriyle olan kombinasyonel özdeşlikleri pek çok araştırmacının dikkatini çekmiştir. Lee ve ark. (2003), Pascal, 1. ve 
2. tip Stirling ve Fibonacci matrislerinden yararlanarak, bu matrisler arasındaki kombinasyonel özdeşlikleri incelemişlerdir. Wang ve Wang (2008), Bell matrisi ve Fibonacci matrisi arasındaki ilişkileri incelemiş, 1. ve 2. tip Stirling matrisleri, Lah matrisi ve genelleştirilmiş Pascal matrisi gibi bazı alt üçgen matrislerin benzeştirilmelerini sağlama üzerine çalışmalar yapmış ve çeşitli özdeşlikler türetmişlerdir. Tang ve ark. (2004) çalışmalarında Pascal matrisini $n \times n$ boyutundaki matrislerin çarpımı olarak ayrıştırılmasının birkaç farklı yoluna değinmiş, bu ayrışımlara dayanarak bir Pascal matrisi ve kompleks bir vektörün çarpımını kolaylaştıracak hızlı algoritmalar üretmişlerdir. Bunun yanı sıra, Edelman ve Strang (1993) çalışmalarında Pascal matrisi ile ilgili temel kavramlara, teoremlere, bu matrisin çeşitli formlarına ve bu formların birbirleriyle olan ilişkilerine yer vermiş, ayrica, Pascal matrislerinin kuvvetlerini, terslerini, logaritmalarını ve özdeğerlerini incelemişlerdir. Cheon ve Kim (2001)'e ait bir çalışmada 1 . ve 2 . tip Stirling sayılarından Pascal tipi matris elde etmeye çalışılmış, bu matrislerin, Pascal matrisi aracılığıyla çarpanlara ayrilabilir olduğu gösterilmiştir. Son olarak, Stirling sayılarının matris gösteriminden bazı iyi tanımlı kombinasyonel özdeşlikler elde edilmiştir.

Bu çalışmada, ilk olarak, Fibonnaci, Pascal, Stirling ve Bell sayıları gibi bazı özel sayı dizileri tanıtılmış, bu sayı dizilerinin elemanları kullanılarak Fibonacci, Pascal, Stirling ve Bell matrisleri oluşturulmuştur. Fibonacci matrislerinin üzerinde özellikle durulmuş ve bu matrislerin, Pascal, Stirling ve Bell matrisleri ile arasındaki ikili bağıntıları incelenmiş, ayrıca bu matrisler aracılığıyla bazı kombinasyonel özdeşlikler ve eşitsizlikler üretilmiştir.

\section{Materyal ve Metot}

Tanım 2.1. Fibonacci sayıları; $F_{0}=0, F_{1}=1$ başlangıç değerleri için lineer rekürans bağıntısı kullanılarak $F_{n}=F_{n-1}+F_{n-2}$ şeklinde tanımlanır (Vajda, 1987).

Tanım 2.2. $F_{n}$; n. Fibonacci sayısı olmak üzere, Fibonacci matrisi, elemanları

$$
f_{i j}=\left\{\begin{array}{ll}
F_{i-j+1}, & i-j+1 \geq 0 \\
0, & i-j+1<0
\end{array} \quad(i, j=0,1,2, \ldots, n)\right.
$$

olacak şekilde $\boldsymbol{F}_{n}=\left(f_{i j}\right)$ ile tanımlanır (Lee, 2000). 
Fibonacci matrisinin açık yazılımı;

$$
\mathbf{F}_{n}=\left(\begin{array}{llllll}
F_{1} & F_{0} & 0 & 0 & \cdots & 0 \\
F_{2} & F_{1} & F_{0} & 0 & \cdots & 0 \\
F_{3} & F_{2} & F_{1} & F_{0} & \cdots & 0 \\
\vdots & \vdots & \vdots & \vdots & \ddots & \vdots \\
F_{n} & F_{n-1} & F_{n-2} & F_{n-3} & \cdots & F_{1}
\end{array}\right)
$$

Tanım 2.3. $\boldsymbol{A}_{n}, n \times n$ alt $\ddot{u} c ̧ g e n$ matrisi, elemanlart

$$
a_{i j}=\left\{\begin{array}{ll}
1, & i=j \\
-1, & i-2 \leq j \leq i-1 \\
0, & \text { diğer durumlarda }
\end{array} \quad(i, j=0,1,2, \ldots, n)\right.
$$

olacak şekilde $\boldsymbol{A}_{n}=\left(a_{i j}\right)$ ile tanımlanır (Wang, 2008).

$\mathbf{A}_{n}$ matrisinin açık yazılımı;

$$
\mathbf{A}_{n}=\left(\begin{array}{cccccc}
1 & 0 & \cdots & \cdots & \cdots & 0 \\
-1 & 1 & 0 & \cdots & \cdots & 0 \\
-1 & -1 & 1 & 0 & \cdots & 0 \\
0 & -1 & -1 & 1 & \ddots & \\
\vdots & \ddots & \ddots & \ddots & \ddots & \\
0 & \cdots & 0 & -1 & -1 & 1
\end{array}\right)
$$

ve $\mathbf{A}_{n}$ matrisinin tersi;

$$
\mathbf{A}_{n}^{-1}=\left(\begin{array}{cccccc}
1 & 0 & \cdots & \cdots & \cdots & 0 \\
1 & 1 & 0 & \cdots & \cdots & 0 \\
2 & 1 & 1 & 0 & \cdots & 0 \\
3 & 2 & 1 & 1 & \ddots & \\
\vdots & \ddots & \ddots & \ddots & \ddots & \\
& \cdots & 3 & 2 & 1 & 1
\end{array}\right)
$$

Bu matris, sütunları Fibonacci dizisinin elemanlarından oluşan Fibonacci matrisidir.

Teorem 2.1. ; $\boldsymbol{F}_{n}$ Fibonacci matrisi olmak üzere, $\boldsymbol{F}_{n}$ 'in tersi, elemanlart

$$
f^{\prime}{ }_{i j}=\left\{\begin{array}{ll}
1, & i=j \\
-1, & i-2 \leq j \leq i-1 \\
0, & \text { diğer durumlarda }
\end{array} \quad(i, j=0,1,2, \ldots, n)\right.
$$

olacak şekilde $\boldsymbol{F}_{n}^{-1}=\left(f^{\prime}{ }_{i j}\right)$ ile tanımlanır (Wang, 2008). 
Tanım 2.4. $0 \leq i, j \leq n-1$ için elemanları $g_{i j}=\left(\begin{array}{c}\mathrm{i}+\mathrm{j} \\ i\end{array}\right)$ olacak şekilde $\boldsymbol{G}_{n}=\left(g_{i j}\right)$ matrisine simetrik Pascal matrisi denir (Edelman, 1993).

Tanım 2.5. $0 \leq i, j \leq n-1$ için elemanları

$$
u_{i j}= \begin{cases}\left(\begin{array}{l}
\mathrm{j} \\
i
\end{array}\right), & j \geq i \\
0, & i>j\end{cases}
$$

olacak şekilde, $\boldsymbol{U}_{n}=\left(u_{i j}\right)$ matrisine üst üçgen Pascal matrisi denir (Edelman, 1993).

Tanım 2.6. $0 \leq i, j \leq n$ için elemanları

$$
p_{i j}= \begin{cases}\left(\begin{array}{l}
\mathrm{i}-1 \\
j-1
\end{array}\right), & i \geq j \\
0, & i<j\end{cases}
$$

olacak şekilde, $\boldsymbol{P}_{n}=\left(p_{i j}\right)$ matrisine alt üçgen Pascal matrisi denir (Cheon, 2001).

Binom katsayıları $C_{r}^{n}=\frac{r !}{n !(r-n) !} ; r=0,1,2, \cdots ; n=0,1,2, \cdots, r$ olmak üzere, simetrik, üst üçgen ve alt üçgen Pascal matrislerinin açık yazılımı aşağıdaki gibidir;

$$
\mathbf{G}_{n}=\left(\begin{array}{llllll}
1 & 1 & 1 & 1 & \cdots & C_{n-1}^{0} \\
1 & 2 & 3 & 4 & \cdots & C_{n}^{1} \\
1 & 3 & 6 & 10 & \cdots & C_{n+1}^{2} \\
1 & 4 & 10 & 20 & \cdots & C_{n+2}^{3} \\
\vdots & \vdots & \vdots & \vdots & \ddots & \vdots \\
C_{n-1}^{0} & C_{n}^{1} & C_{n+1}^{2} & C_{n+2}^{3} & \cdots & C_{2 n-2}^{n-1}
\end{array}\right)
$$

$\mathbf{U}_{n}=\left(\begin{array}{llllll}1 & 1 & 1 & 1 & \cdots & C_{n-1}^{0} \\ 0 & 1 & 2 & 3 & \cdots & C_{n-1}^{1} \\ 0 & 0 & 1 & 3 & \cdots & C_{n-1}^{2} \\ 0 & 0 & 0 & 1 & \cdots & C_{n-1}^{3} \\ \vdots & \vdots & \vdots & \vdots & \ddots & \vdots \\ 0 & 0 & 0 & 0 & \cdots & C_{n-1}^{n-1}\end{array}\right)$ ve $\mathbf{P}_{n}=\left(\begin{array}{llllll}1 & 0 & 0 & 0 & \cdots & 0 \\ 1 & 1 & 0 & 0 & \cdots & 0 \\ 1 & 2 & 1 & 0 & \cdots & 0 \\ 1 & 3 & 3 & 1 & \cdots & 0 \\ \vdots & \vdots & \vdots & \vdots & \ddots & \vdots \\ C_{n}^{0} & C_{n}^{1} & C_{n}^{2} & C_{n}^{3} & \cdots & C_{n}^{n}\end{array}\right)$

$\mathbf{G}_{n}$; simetrik Pascal matrisini, $\mathbf{U}_{n}$; üst üçgen Pascal matrisini ve $\mathbf{P}_{n}$; alt üçgen Pascal matrisini göstermek üzere, Pascal matrislerinin bazı özellikleri aşağıdaki gibidir.

- $\operatorname{det}\left(\mathbf{G}_{n}\right)=1, \operatorname{det}\left(\mathbf{U}_{n}\right)=1, \operatorname{det}\left(\mathbf{P}_{n}\right)=1$,

- $\mathbf{U}_{n}=\mathbf{P}_{n}^{T}$ ve $\mathbf{P}_{n}=\mathbf{U}_{n}^{T}$, 
- $\mathbf{G}_{n}=\mathbf{U}_{n} \mathbf{P}_{n}$ ve $\mathbf{G}_{n}=\mathbf{P}_{n} \mathbf{U}_{n}($ Edelman, 1993).

Tanım 2.7. $n$ ve $k$ pozitif tam sayıları için 1. ve 2. tip Stirling sayılarl;

$$
[x]_{n}= \begin{cases}x(x-1) \cdots(x-n+1,) & n \geq 1 \\ 1, & n=0\end{cases}
$$

olmak üzere $[x]_{n}=\sum_{k=0}^{n}(-1)^{n-k} S(n, k) x^{k}$ ve $x^{n}=\sum_{k=0}^{n} S(n, k)[x]_{k}$ seri açılımındaki $x$ 'in katsayllarl sirasiyla $s(n, k)$ ve $S(n, k)$ olarak tanımlanır (Cheon, 2001).

$n$ ve $k$ pozitif tam say1ları ve $s(n, 0)=s(0, k)=S(n, 0)=S(0, k)=[0]_{k}=0$ ve $s(0,0)=$ $S(0,0)=1$ için $s(n, k), S(n, k)$ ve $[n]_{k}$;

$$
\begin{aligned}
s(n, k) & =s(n-1, k-1)+(n-1) s(n-1, k), \\
S(n, k) & =S(n-1, k-1)+k S(n-1, k), \\
{[n]_{k} } & =[n-1]_{k}+k[n-1]_{k-1}
\end{aligned}
$$

ile verilen Pascal tipi rekürans bağıntılarını sağlarlar ve buna ek olarak;

$$
S(n, k)=\sum_{\ell=k-1}^{n-1}\left(\begin{array}{c}
\mathrm{n}-1 \\
\ell
\end{array}\right) S(\ell, k-1) \quad(\text { Cheon, 2001). }
$$

Tanım 2.8. $s(i, j)$ ve $S(i, j)$ strastyla 1. ve 2. tip Stirling sayllarl ve elemanlart

$$
s_{i j}=\left\{\begin{array}{ll}
s(i, j), & i \geq j \\
0, & \text { diğ er durumlarda }
\end{array} \quad(i, j=0,1,2, \ldots, n)\right.
$$

ve

$$
S_{i j}=\left\{\begin{array}{ll}
S(i, j), & i \geq j \\
0, & \text { dĭg er durumlarda }
\end{array} \quad(i, j=0,1,2, \ldots, n)\right.
$$

olacak şekilde $n \times n$ boyutlu 1. ve 2. tip Stirling matrisleri $\boldsymbol{S}_{n}(1)=\left(s_{i j}\right)$ ve $\boldsymbol{S}_{n}(2)=\left(S_{i j}\right)$ olarak tanımlanir (Cheon, 2001).

Tanım 2.9. Bell sayıları, $B_{0}=1$ başlangıç değeri için lineer rekürans bağıntıları kullanılarak

şeklinde tanımlanır (Wang, 2008).

$$
B_{n}=\sum_{k=0}^{n-1} B_{k}\left(\begin{array}{c}
n-1 \\
k
\end{array}\right)
$$

Bell sayıları arasında pek çok bağıntı vardır, bunlardan bazıları aşağıda verilmiştir:

- Her bir Bell sayıs1 2. tip Stirling sayıları toplamıdır; $B_{n}=\sum_{k=0}^{n} S(n, k)$. 
- Bell sayıları $f(x)=e^{e^{x}}$ fonksiyonunun Mc Lauren açılımının katsayılarıdır;

$$
e^{e^{x}}=e\left(1+\frac{x}{1}+\frac{2 x^{2}}{2 !}+\frac{5 x^{3}}{3 !}+\cdots\right) \text {. }
$$

Tanım 2.10. $B_{n} ; n$. Bell saylst ve elemanlart

$$
b_{i j}= \begin{cases}B_{i-j}, & i-j \geq 0 \\ 0, & i-j<0\end{cases}
$$

olacak şekilde Bell matrisi $\boldsymbol{B}_{n}=\left(b_{i j}\right)$ olarak tanımlanır (Wang, 2008).

Bell matrisinin açık yazılımı;

$$
\boldsymbol{B}_{n}=\left(\begin{array}{llllll}
B_{0} & 0 & 0 & 0 & \cdots & 0 \\
B_{1} & B_{0} & 0 & 0 & \cdots & 0 \\
B_{2} & B_{1} & B_{0} & 0 & \cdots & 0 \\
\vdots & \vdots & \vdots & \vdots & \ddots & \vdots \\
B_{n-1} & B_{n-2} & B_{n-3} & B_{n-4} & \cdots & B_{0}
\end{array}\right) .
$$

\section{Araştırma Sonuçları ve Tartışma}

Çalışmanın bu kısmında, Materyal ve Metot bölümünde tanımlanan Fibonacci matrisinin, sırasıyla Pascal, Stirling ve Bell matrisleri ile arasındaki kombinasyonel özdeşliklerin elde edilişi üzerinde durulmuştur.

\subsection{Fibonacci Matrisi ve Pascal Matrisi Arasındaki Özdeşlikler}

Tanım 3.1. $\boldsymbol{L}_{\boldsymbol{n}}=\left(l_{i j}\right)$ matrisi, elemanlart

$$
l_{i j}=\left(\begin{array}{l}
i-1 \\
j-1
\end{array}\right)-\left(\begin{array}{l}
i-2 \\
j-1
\end{array}\right)-\left(\begin{array}{l}
i-3 \\
j-1
\end{array}\right)
$$

olacak şekilde, $l_{11}=1, j \geq 2$ için $l_{1 j}=0 ; l_{21}=0, l_{22}=1, j \geq 3$ için $l_{2 j}=0 ; i \geq 3$ için $l_{i 1}=-1$ ve $i, j \geq 2$ için $l_{i j}=l_{i-1, j-1}+l_{i-1, j}$ ile tanımlanır (Lee, 2003).

Teorem 3.1. $\boldsymbol{P}_{n} ;$ Pascal matrisi, $\boldsymbol{F}_{n} ;$ Fibonacci matrisi, $\boldsymbol{L}_{n}$; elemanları Tanım 3.1 ile verilen matris olmak üzere

$$
\mathbf{P}_{n}=\mathbf{F}_{n} \mathbf{L}_{n} \quad(\text { Lee, 2003). }
$$

Íspat. $\mathbf{F}_{n}^{-1} \mathbf{P}_{n}=\mathbf{L}_{n}$ olduğunu göstermemiz yeterlidir. Teorem 2.1'den Fibonacci matrisinin tersinin varolduğunu ve $\mathbf{F}_{n}^{-1}=\left(f^{\prime}{ }_{i j}\right)$ şeklinde tanımlandığını biliyoruz. $j \geq 2$ için $f^{\prime}{ }_{1 j}=0$, $f_{11}^{\prime} p_{11}=1$ ve $l_{11}=1=\sum_{k=1}^{n} f_{1 k}^{\prime} p_{k 1} . j \geq 2$ için $p_{1 j}=0 \quad$ ve $f_{1 j}^{\prime}=0, j \geq 2$ için $\sum_{k=1}^{n} f_{1 k}^{\prime} p_{k j}=0=l_{1 j} . j \geq 3$ için $f^{\prime}{ }_{2 j}=0 ; f^{\prime}{ }_{21}=-1, f^{\prime}{ }_{22}=1, \sum_{k=1}^{n} f^{\prime}{ }_{2 k} p_{k 1}=l_{21}$. 
Öte yandan, Teorem 2.1 'den $i=3,4, \cdots, n$ için $\sum_{k=1}^{n} f^{\prime}{ }_{i k} p_{k 1}=l_{i 1} . i \geq 3$ ve $j \geq 2$ için, Teorem 2.1 'den ve $l_{i j}$ 'in rekürans bağıntısından $\sum_{k=1}^{n} f_{i k}^{\prime} p_{k j}=l_{i j}$. Dolayısıyla $\mathbf{F}_{n}^{-1} \mathbf{P}_{n}=$ $\mathbf{L}_{n}$.

Sonuç 3.1.1. $1 \leq r \leq n$ için,

$$
\left(\begin{array}{l}
n-1 \\
r-1
\end{array}\right)=\sum_{k=r}^{n} F_{n-k+1} \frac{(k-3) !\left(r(k-1)-2(r-1)-(k-r)^{2}\right)}{(r-1) !(k-r) !}
$$

Özellikle, $r=1$ için $F_{1}+F_{2}+\cdots+F_{n-2}=F_{n}-1 \quad($ Lee, 2003).

Íspat. $F_{1}=F_{2}=1, i \leq j+1$ için $l_{i j}=0$ ve $\mathbf{P}_{n}=\mathbf{F}_{n} \mathbf{L}_{n}$ çarpımından;

$$
\left(\begin{array}{c}
\mathrm{n}-1 \\
r-1
\end{array}\right)=p_{n r}=\sum_{k=1}^{n} f_{n k} l_{k r}=f_{n 1} l_{1 r}+f_{n 2} l_{2 r}+\cdots+f_{n, n-2} l_{n-2, r}+f_{n, n-1} l_{n-1, r}+f_{n n} l_{n r} .
$$

Tanım 2.2'den yararlanarak özdeşliği düzenlersek;

$$
\left(\begin{array}{c}
\mathrm{n}-1 \\
r-1
\end{array}\right)=p_{n r}=\sum_{k=1}^{n} F_{n-k+1} l_{k r}=F_{n} l_{1 r}+F_{n-1} l_{2 r}+\cdots+F_{3} l_{n-2, r}+F_{2} l_{n-1, r}+F_{1} l_{n r}
$$

$l_{r r}=1, l_{r+1, r}=r-1$ ve $k \geq r+2$ için;

$$
\begin{aligned}
l_{k r}=\left(\begin{array}{l}
\mathrm{k}-1 \\
r-1
\end{array}\right)-\left(\begin{array}{l}
\mathrm{k}-2 \\
r-1
\end{array}\right)-\left(\begin{array}{l}
\mathrm{k}-3 \\
r-1
\end{array}\right) & =\frac{(k-1) !}{(k-r) !(r-1) !}-\frac{(k-2) !}{(k-r-1) !(r-1) !}-\frac{(k-3) !}{(k-r-2) !(r-1) !} \\
& =\frac{(k-3) !\left(r(k-1)-2(r-1)-(k-r)^{2}\right)}{(r-1) !(k-r) !} .
\end{aligned}
$$

Öte yandan, özellikle $r=1$ olduğu zaman $l_{11}=1, l_{21}=0$ ve $i=3,4, \cdots, n$ için $l_{i 1}=-1$.

Dolayısıyla;

$$
\begin{aligned}
& 1=p_{n 1}=F_{n} l_{11}+F_{n-1} l_{21}+F_{n-2} l_{31}+\cdots+F_{3} l_{n-2,1}+F_{2} l_{n-1,1}+F_{1} l_{n 1}, \\
& 1=p_{n 1}=F_{n} 1+F_{n-1} 0+F_{n-2}(-1)+\cdots+F_{3}(-1)+F_{2}(-1)+F_{1}(-1) .
\end{aligned}
$$

Sonuç olarak $F_{1}+F_{2}+F_{3}+\cdots+F_{n-2}=F_{n}-1$.

Tanım 3.2. $\boldsymbol{L}_{n}$ matrisinin tersi, elemanlart

$$
l_{i j}^{\prime}=\sum_{k=j}^{i}(-1)^{k}\left(\begin{array}{c}
i-1 \\
k-1
\end{array}\right) F_{k-j+1}
$$

olacak şekilde $\mathbf{L}_{n}^{-1}=\left(l^{\prime}{ }_{i j}\right)$ olarak tanımlanır (Lee, 2003).

$j \geq 2$ için $l_{i j}^{\prime}=l_{i-1, j-1}^{\prime}-l_{i-1, j}^{\prime} \quad$ ve $i \geq 3$ için $l_{i 1}^{\prime}=(-1)^{i+1} F_{i-2} . \quad \boldsymbol{F}_{n}=\boldsymbol{P}_{n} \boldsymbol{L}_{n}^{-1}$ eşitliğinden

$$
F_{n}=\sum_{k=2}^{n+1}(-1)^{k}\left(\begin{array}{c}
n \\
k-1
\end{array}\right) F_{k-1} \quad(\text { Lee }, 2003)
$$


Ayrıca, (8) ve (9)'dan aşağıdaki sonuç elde edilir.

Sonuç 3.1.2. $F_{n}$, n. Fibonacci sayısı olmak üzere $n \geq 3$ için;

$$
F_{n}=1+\sum_{j=3}^{n}(-1)^{j+1}\left(\begin{array}{c}
n-1 \\
j-1
\end{array}\right) F_{j-2}=2^{n-2}-\sum_{j=1}^{n-3} F_{k} 2^{n-k-3} \quad(\text { Lee }, 2003) .
$$

Ispat. $\mathbf{F}_{n}=\left(f_{i j}\right)=\mathbf{P}_{n} \mathbf{L}_{n}^{-1}$ ve $f_{n 1}=F_{n}$ için $F_{n}=\sum_{j=1}^{n} p_{n j} l_{j 1}^{\prime}$. Ayrıca, $l_{11}^{\prime}=1, l_{21}^{\prime}=0$ ve $l_{j 1}^{\prime}=(-1)^{j+1} F_{j-2}$ için,

$$
\begin{aligned}
F_{n} & =\sum_{j=1}^{n} p_{n j} l_{j 1}^{\prime}=p_{n 1} l_{11}^{\prime}+p_{n 2} l_{21}^{\prime}+\sum_{j=3}^{n} p_{n j} l_{j 1}^{\prime}=1+\sum_{j=3}^{n} p_{n j} l_{j 1}^{\prime}, \\
& =p_{n 1}+\sum_{j=3}^{n}(-1)^{j+1} p_{n j} F_{j-2}=1+\sum_{j=3}^{n}(-1)^{j+1}\left(\begin{array}{c}
\mathrm{n}-1 \\
j-1
\end{array}\right) F_{j-2} .
\end{aligned}
$$

Şimdi, ikinci özdeşliği ispatlayalım; $E_{n}=(1,1,1, \cdots, 1)^{T}$ ve Teorem 3.1'den $\mathbf{P}_{n} E_{n}=\mathbf{F}_{n} \mathbf{L}_{n} E_{n}$. Öncelikle $\mathbf{L}_{n}$ ve $E_{n}$ çarpımından $n \geq 4$ için $l_{n 1}+l_{n 2}+l_{n 3}+\cdots+l_{n n}=2^{n-3}$. Bulunan ifade $\mathbf{F}_{n}$ ile çarpıldığında, $n \geq 4$ için

$\mathbf{F}_{n} \mathbf{L}_{n} E_{n}=F_{n}+F_{n-1}+F_{n-2}+\sum_{k=4}^{n} F_{k-3} 2^{n-k+1}=F_{n}+F_{n-1}+F_{n-2}+\sum_{k=1}^{n-3} F_{k} 2^{n-k-2}$.

Öte yandan özdeşliğin sol tarafı $\mathbf{P}_{n} E_{n}=\left(1,2,4,8, \cdots, \sum_{k=0}^{n-1}\left(\begin{array}{c}\mathrm{n}-1 \\ k\end{array}\right)\right)^{T}$. Binom katsayılar arasındaki ilişkiden $\quad \sum_{k=0}^{n-1}\left(\begin{array}{c}n-1 \\ k\end{array}\right)=2^{n-1} \quad$ olduğundan $\quad \mathbf{P}_{n} E_{n}=\left(1,2,4,8, \cdots, 2^{n-1}\right)^{T}$. Dolayısıyla,

$n \geq 3$ için $2^{n-1}=F_{n}+F_{n-1}+F_{n-2}+\sum_{k=1}^{n-3} F_{k} 2^{n-k-2}$.

Sonuç 3.1.1, Sonuç 3.1.2 ve $\sum_{i=0}^{n}\left(\begin{array}{c}\mathrm{n}-\mathrm{i} \\ i\end{array}\right)=F_{n+1}$ kombinasyonel özdeşliğinden $\left\{F_{n}\right\}$ Fibonacci dizisinin ilk $n$ teriminin toplamı;

$$
F_{1}+F_{2}+\cdots+F_{n}=\sum_{j=3}^{n+2}(-1)^{j+1}\left(\begin{array}{c}
\mathrm{n}+1 \\
j-1
\end{array}\right) F_{j-2}=2^{n}-\sum_{k=1}^{n-1} F_{k} 2^{n-k-1}-1 .
$$

\subsection{Fibonacci Matrisi ve 2. Stirling Matrisi Arasındaki Özdeşlikler}

Tanım 3.3. $\boldsymbol{M}_{n}=\left(m_{i j}\right)$; elemanlart

$$
m_{i j}=S(i, j)-S(i-1, j)-S(i-2, j)
$$

olmak üzere, $m_{11}=1, j \geq 2$ için $m_{1 j}=0 ; m_{21}=0, m_{22}=1, j \geq 3$ için $m_{2 j}=0 ; i \geq 3$ için $m_{i 1}=-1 ; i, j \geq 2$ için $m_{i j}=m_{i-1, j-1}+j m_{i-1, j}$ (Lee, 2003).

Teorem 3.2. $\boldsymbol{M}_{n}$; elemanları Tanım 3.3 ile verilen $n \times n$ matris, $\boldsymbol{S}_{n}(2) ; 2$. tip Stirling matrisi ve $\boldsymbol{F}_{n}$; Fibonacci matrisi olmak üzere

$$
\boldsymbol{S}_{n}(2)=\boldsymbol{F}_{n} \boldsymbol{M}_{n} \quad(\text { Lee, } 2003)
$$


Íspat. $\mathbf{F}_{n}^{-1} \mathbf{S}_{n}(2)=\mathbf{M}_{n}$ olduğunu göstermemiz yeterlidir. $\mathbf{F}_{n}^{-1}=\left(f_{i j}^{\prime}\right), \mathbf{F}_{n}{ }^{\prime}$ in tersi olmak üzere $j \geq 2$ için $f_{1 j}^{\prime}=0, f_{11}^{\prime} S_{11}=1=m_{11} ; j \geq 2$ için $S_{1 j}=0$ ve $f_{1 j}^{\prime}=0, \sum_{k=1}^{n} f_{1 k}^{\prime} S_{k j}=$ $0=m_{1 j} . j \geq 3$ için $f^{\prime}{ }_{2 j}=0 ; f^{\prime}{ }_{21}=-1$ ve $f^{\prime}{ }_{22}=1, \sum_{k=1}^{n} f_{2 k}^{\prime} S_{k 1}=0=m_{21}$. (1)'den $i=$ $3,4, \cdots, n$ için $\sum_{k=1}^{n} f^{\prime}{ }_{i k} S_{k 1}=m_{i 1}$. Öte yandan, $i \geq 3$ ve $j \geq 2$ için (4) ve (10)'dan $\sum_{k=1}^{n} f_{i k}^{\prime} S_{k j}=m_{i j}$. Sonuç olarak $\mathbf{F}_{n}^{-1} \mathbf{S}_{n}(2)=\mathbf{M}_{n}$, yani $\mathbf{S}_{n}(2)=\mathbf{F}_{n} \mathbf{M}_{n} . \quad S_{n k}=S(n, k)=$ $\sum_{r=1}^{n} f_{n r} m_{r k}$ ve $i \geq 3$ için

$$
m_{i k}=\frac{1}{k !} \sum_{0 \leq \ell \leq k}(-1)^{\ell}\left(\begin{array}{l}
k \\
\ell
\end{array}\right)\left((k-\ell)^{i}-(k-\ell)^{i-1}-(k-\ell)^{i-2}\right),
$$

olduğunda aşağıdaki sonucu elde ederiz.

Sonuç 3.2.1. $1 \leq k \leq n$ için;

$S(n, k)=\sum_{i=k}^{n} F_{n-i+1}\left(\frac{1}{k !} \sum_{0 \leq \ell \leq k}(-1)^{\ell}\left(\begin{array}{c}\mathrm{k} \\ \ell\end{array}\right)\left((k-\ell)^{i}-(k-\ell)^{i-1}-(k-\ell)^{i-2}\right)\right)$

(Lee,2003).

Lemma 3.3. $\boldsymbol{S}_{n-1}(2) ;(n-1) \times(n-1)$ boyutlu 2. tip Stirling matrisi, $\boldsymbol{L}_{n}$; elemanlart Tanım3.1 ile verilen matris ve $\boldsymbol{M}_{n}$; elemanları Tanım 3.3 ile verilen matris olmak üzere

$$
\boldsymbol{M}_{n}=\boldsymbol{L}_{n}\left([1] \oplus \boldsymbol{S}_{n-1}(2)\right) \quad(\text { Lee }, 2003) .
$$

Ispat. $\mathbf{D}_{n}=\left(d_{i j}\right)=\mathbf{L}_{n}\left([1] \oplus \mathbf{S}_{n-1}(2)\right)$ olsun, (6) ve (10)' dan $l_{11}=1=m_{11}, l_{21}=0=$ $m_{21}$ ve $l_{22}=S(1,1)=1=m_{22} ; i=1,2$ için $d_{i j}=m_{i j} . i \geq 3$ için

$$
d_{i j}=\sum_{k=j-1}^{i-1}\left[\left(\begin{array}{c}
\mathrm{i}-1 \\
k
\end{array}\right) S(k, j-1)-\left(\begin{array}{c}
\mathrm{i}-2 \\
k
\end{array}\right) S(k, j-1)-\left(\begin{array}{c}
\mathrm{i}-3 \\
k
\end{array}\right) S(k, j-1)\right]
$$

ve (2)'den $d_{i j}=S(i, j)-S(i-1, j)-S(i-2, j)=m_{i j}$.

Dolayısıyla, $\mathbf{M}_{n}=\mathbf{L}_{n}\left([1] \oplus \mathbf{S}_{n-1}(2)\right)$.

Aşağıdaki sonuç Lemma 3.3'ün doğrudan sonucudur.

Sonuç 3.3.1. $\boldsymbol{S}_{n}(2) ;$ 2. Stirling matrisi, $\boldsymbol{F}_{n}$; Fibonacci matrisi, $\boldsymbol{L}_{n}$; elemanları Tanım 3.1 ile verilen matris olmak üzere $n \geq 2$ için $\boldsymbol{S}_{n}(2)=\boldsymbol{F}_{n} \boldsymbol{L}_{n}\left([1] \oplus \boldsymbol{S}_{n-1}(2)\right)$ (Lee, 2003).

Ispat. Teorem 3.1'den $\mathbf{F}_{n} \mathbf{L}_{n}=\mathbf{P}_{n}$. Burada $n \times n$ Pascal matrisi olmak üzere $\mathbf{S}_{n}(2)=$ $\mathbf{P}_{n}\left([1] \oplus \mathbf{S}_{n-1}(2)\right) . i \geq j \geq 1$ koşuluyla $(i, j)$ başlangiç değeri için [1] $\oplus \mathbf{S}_{n-1}(2)$; $S(i-$ $1, j-1)$. Matris çarpımı tanımından ve (2)'den

$\mathbf{P}_{n}\left([1] \oplus \mathbf{S}_{n-1}(2)\right)_{i j}=\sum_{\ell=j-1}^{i-1} p_{i, \ell+1} S(\ell, j-1)=\sum_{\ell=j-1}^{i-1}\left(\begin{array}{c}\mathrm{i}-1 \\ \ell\end{array}\right) S(\ell, j-1)=S(i, j)=$ $\mathbf{S}_{n}(2)$.

Tanım 3.4. $\boldsymbol{P}_{k} ; k \times k$ Pascal matrisi ve $\boldsymbol{I}_{n-k} ;(n-k)$. dereceden birim matris olmak üzere, $\overline{\boldsymbol{P}}_{k} ; n \times n$ matris $\overline{\boldsymbol{P}}_{k}=\boldsymbol{I}_{n-k} \oplus \boldsymbol{P}_{k}$ ile tanimlanir. Buradan $\overline{\boldsymbol{P}}_{n}=\boldsymbol{P}_{n}$ ve $\overline{\boldsymbol{P}}_{1} \boldsymbol{I}_{n}($ Lee, 2003$)$. 
Sonuç 3.3.2. $\boldsymbol{S}_{n}(2) ; 2$. Stirling matrisi ve $\overline{\boldsymbol{P}}_{k}$; Tanım 3.4 ile tanımlanan Pascal matrisi olmak üzere $\boldsymbol{S}_{n}(2), \overline{\boldsymbol{P}}_{k}$ aracılı̆̆lyla $\boldsymbol{S}_{n}(2)=\overline{\boldsymbol{P}}_{n} \overline{\boldsymbol{P}}_{n-1} \cdots \overline{\boldsymbol{P}}_{2} \overline{\boldsymbol{P}}_{1}$ şeklinde üretilir.

$\mathbf{F}_{k} ; k \times k$ Pascal matrisi ve $\mathbf{L}_{k}$; elemanları Tanım 3.1 ile verilen $k \times k$ matris olmak üzere $n \times n, \overline{\mathbf{F}_{k} \mathbf{L}_{k}}$ matrisi, $\overline{\mathbf{F}_{k} \mathbf{L}_{k}}=\mathbf{I}_{n-k} \oplus \mathbf{F}_{k} \mathbf{L}_{k}$ ile tanımlanır. $\overline{\mathbf{F}_{n} \mathbf{L}_{n}}=\mathbf{F}_{n} \mathbf{L}_{n}$ ve $\overline{\mathbf{F}_{1} \mathbf{L}_{1}}=\mathbf{I}_{n}$ için,

$$
\mathbf{S}_{n}(2)=\left(\overline{\mathbf{F}_{n} \mathbf{L}_{n}}\right)\left(\overline{\mathbf{F}_{n-1} \mathbf{L}_{n-1}}\right) \cdots \overline{\mathbf{F}_{1} \mathbf{L}_{1}} \quad(\text { Lee, 2003) }
$$

\section{3 . Fibonacci Matrisi ve 1. Stirling Matrisi Arasındaki Özdeşlikler}

Tanım 3.5. $\boldsymbol{Q}_{n}=\left(q_{i j}\right)$; elemanlart

$$
q_{i j}=s(i, j)-s(i, j+1)-s(i, j+2)
$$

olmak üzere, $q_{11}=1, j \geq 2$ için $q_{1 j}=0 ; q_{21}=0, q_{22}=1$ ve $j \geq 3$ için $q_{2 j}=0 ; i, j \geq 2$ için $q_{i j}=q_{i-1, j-1}+(i-1) q_{i-1, j}($ Lee, 2003).

Teorem 3.4. $\boldsymbol{Q}_{n}$; elemanları Tanım 3.5 ile verilen $n \times n$ matris, $\boldsymbol{S}_{n}(1)$; 1 . tip Stirling matrisi ve $\boldsymbol{F}_{n}$; Fibonacci matrisi olmak üzere,

$$
\mathbf{S}_{\mathrm{n}}(1)=\mathbf{Q}_{\mathrm{n}} \mathbf{F}_{\mathrm{n}}(\text { Lee, 2003). }
$$

Ispat. $\mathbf{S}_{n}(1) \mathbf{F}_{n}^{-1}=\mathbf{Q}_{n}$ olduğunu göstermemiz yeterlidir. $\mathbf{F}_{n}^{-1}=\left(f_{i j}^{\prime}\right), \mathbf{F}_{n}{ }^{\prime}$ in tersi olmak üzere $i \geq 1$ ve $j=1,2, \cdots, n-2$ için $\sum_{k=1}^{n} s_{i k} f^{\prime}{ }_{k j}=q_{i j} . j=n-1$ için $\sum_{k=1}^{n} s_{i k} f^{\prime}{ }_{k, n-1}=0=$ $q_{i, n-1}$ ve $j=n$ için $\sum_{k=1}^{n} s_{i k} f_{k n}^{\prime}=s_{i n}=s(i, n)=q_{i n}$. Sonuç olarak $\mathbf{S}_{n}(1) \mathbf{F}_{n}^{-1}=\mathbf{Q}_{n}$, yani $\mathbf{S}_{n}(1)=\mathbf{Q}_{n} \mathbf{F}_{n}$.

Teorem 3.4'den $\mathbf{S}_{n}(1)=\mathbf{Q}_{n} \mathbf{F}_{n}$ olduğunu biliyoruz. $\mathbf{S}_{n}(1) E_{n}=\mathbf{Q}_{n} \mathbf{F}_{n} E_{n}$ için

$$
n !=\sum_{k=1}^{n}(s(n, k)-s(n, k+1)-s(n, k+2))\left(F_{k+2}-1\right) .
$$

$\mathbf{S}_{n}^{-1}(1)$; 1. Stirling matrisinin tersi, $\mathbf{S}_{n}^{-1}(2) ; 2$. Stirling matrisinin tersi, ve $\mathbf{P}_{n}^{-1}$; Pascal matrisinin tersidir ve $\mathbf{S}_{n}^{-1}(2)=\left[(-1)^{i-j} S_{i j}\right]$ ve $\mathbf{S}_{n}^{-1}(1)=\left[(-1)^{i-j} S_{i j}\right]$. Buradan, $\mathbf{P}_{n}^{-1}=$ $\left[(-1)^{i-j}\left(\begin{array}{l}\mathrm{i}-1 \\ j-1\end{array}\right)\right]$ için $\mathbf{S}_{n}(1)=\left([1] \oplus \mathbf{S}_{n-1}(1)\right) \mathbf{P}_{n}$. Yani, $\mathbf{P}_{n}=\mathbf{S}_{n}(2)\left([1] \oplus \mathbf{S}_{n-1}^{-1}(2)\right)$ ve $\mathbf{P}_{n}=\left([1] \oplus \mathbf{S}_{n-1}^{-1}(1)\right) \mathbf{S}_{n}(1)$.

Buradan yola çıkarak aşağıda verilen teorem elde edilir.

Teorem 3.5. $\boldsymbol{F}_{n}$; Fibonacci matrisi, $\boldsymbol{S}_{n}(1) ; 1$. tip Stirling matrisi ve $\boldsymbol{L}_{n}$; elemanlarl Tanım 3.1 ile verilen matris olmak üzere

$$
\boldsymbol{S}_{n}(1)=\left([1] \oplus \boldsymbol{S}_{n-1}(1)\right) \boldsymbol{F}_{n} \boldsymbol{L}_{n}=\overline{\boldsymbol{P}}_{1} \overline{\boldsymbol{P}}_{2} \cdots \overline{\boldsymbol{P}}_{n} \quad(\text { Lee }, 2003) .
$$

Aşağıdaki sonuç, Teorem 3.4 ve Teorem 3.5'den elde edilir.

Sonuç 3.5.1 $1 \leq k \leq n$ için $s(n, k)=\sum_{\ell=k-1}^{n-1} s(n-1, \ell)\left(\begin{array}{c}\ell \\ k-1\end{array}\right)$. 


\subsection{Fibonacci Matrisi ve Bell Matrisi Arasındaki Özdeşlikler}

Tanım 3.6. $\quad b_{i j}$; Tanım 2.10 ile verilen Bell matrisinin elemanları olmak üzere, $i, j=$ $1,2, \cdots, n$ için elemanları

$$
\begin{aligned}
& q_{i j}=b_{i j}-b_{i-1, j}-b_{i-2, j} \\
& z_{i j}=b_{i j}-b_{i, j+1}-b_{i, j+2}
\end{aligned}
$$

olacak şekilde $\boldsymbol{N}_{n}=\left(q_{i j}\right)$ ve $\boldsymbol{R}_{n}=\left(z_{i j}\right)$ matrisleri tanımlanır (Wang, 2008).

Lemma 3.6 $\boldsymbol{B}_{n}$; Bell matrisi, $\boldsymbol{A}_{n}$; Tanım 2.3 ile verilen tersi Fibonacci matrisini veren matris ve $\boldsymbol{N}_{n}$ ile $\boldsymbol{R}_{n}$; Tanım 3.6 ile verilen matrisler olmak üzere

$$
\mathbf{A}_{n} \mathbf{B}_{n}=\mathbf{N}_{n} \quad \text { ve } \quad \mathbf{B}_{n} \mathbf{A}_{n}=\mathbf{R}_{n} \quad \text { (Wang, 2008). }
$$

Ispat. Öncelikle Tanım 3.6 ile verilen $\mathbf{N}_{\mathrm{n}}$ matrisinin elemanlarını (13) bağıntısı ile belirleyelim. $q_{11}=b_{11}, j \geq 2$ için $q_{1 j}=0 ; q_{21}=b_{21}-b_{11}, q_{22}=b_{22}$ ve $j \geq 3$ için $q_{2 j}=$ 0 . Benzer şekilde $\mathbf{R}_{\mathrm{n}}$ matrisinin elemanları (14) bağıntısı ile belirlenebilir. Şimdi $\mathbf{A}_{\mathrm{n}} \mathbf{B}_{\mathrm{n}}=\mathbf{N}_{\mathrm{n}}$ denklemini ele alalım. Tanım 2.3'ten $\mathbf{A}_{\mathrm{n}}=\mathbf{F}_{\mathrm{n}}^{-1}=\left(\mathrm{f}_{\mathrm{ij}}^{\prime}\right)$ olduğu bilinmektedir ve $\mathbf{F}_{\mathrm{n}}^{-1} \mathbf{B}_{\mathrm{n}}=\mathbf{N}_{\mathrm{n}}$ olduğunu göstermemiz yeterlidir. $\mathrm{f}^{\prime}{ }_{11}=1, \sum_{\mathrm{k}=1}^{\mathrm{n}} \mathrm{f}^{\prime}{ }_{1 \mathrm{k}} \mathrm{b}_{\mathrm{k} 1}=\mathrm{f}^{\prime}{ }_{11} \mathrm{~b}_{11}=\mathrm{b}_{11}=\mathrm{q}_{11}$ ve $\mathrm{j} \geq 2$ için $\mathrm{f}_{1 \mathrm{j}}^{\prime}=\mathrm{b}_{1 \mathrm{j}}=0, \sum_{\mathrm{k}=1}^{\mathrm{n}} \mathrm{f}_{1 \mathrm{k}}^{\prime} \mathrm{b}_{\mathrm{kj}}=\mathrm{f}_{11}^{\prime} \mathrm{b}_{1 \mathrm{j}}=0=\mathrm{q}_{1 \mathrm{j}} . \mathrm{f}_{21}^{\prime}=-1, \mathrm{f}_{22}^{\prime}=1, \sum_{\mathrm{k}=1}^{\mathrm{n}} \mathrm{f}_{2 \mathrm{k}}^{\prime} \mathrm{b}_{\mathrm{k} 1}=\mathrm{f}_{21}^{\prime} \mathrm{b}_{11}+$ $\mathrm{f}_{22}^{\prime} \mathrm{b}_{21}=\mathrm{b}_{21}-\mathrm{b}_{11}=\mathrm{q}_{21}, \sum_{\mathrm{k}=1}^{\mathrm{n}} \mathrm{f}_{2 \mathrm{k}}^{\prime} \mathrm{b}_{\mathrm{k} 2}=\mathrm{f}_{21}^{\prime} \mathrm{b}_{12}+\mathrm{f}_{22}^{\prime} \mathrm{b}_{22}=\mathrm{b}_{22}=\mathrm{q}_{22}$ ve $\mathrm{j} \geq 3$ için $\mathrm{f}_{2 \mathrm{j}}^{\prime}=0$, ve $\sum_{\mathrm{k}=1}^{\mathrm{n}} \mathrm{f}_{2 \mathrm{k}}^{\prime} \mathrm{b}_{\mathrm{kj}}=\mathrm{f}_{21}^{\prime} \mathrm{b}_{1 \mathrm{j}}+\mathrm{f}_{22}^{\prime} \mathrm{b}_{2 \mathrm{j}}=0=\mathrm{q}_{2 \mathrm{j}}$. Öte yandan $\mathrm{i} \geq 3$ için (1) ve (5)'den $\sum_{\mathrm{k}=1}^{\mathrm{n}} \mathrm{f}_{\mathrm{ik}}^{\prime} \mathrm{b}_{\mathrm{kj}}=\mathrm{f}_{\mathrm{ii}}^{\prime} \mathrm{b}_{\mathrm{ij}}+\mathrm{f}_{\mathrm{i}, \mathrm{i}-1}^{\prime} \mathrm{b}_{\mathrm{i}-1, \mathrm{j}}+\mathrm{f}_{\mathrm{i}, \mathrm{i}-2}^{\prime} \mathrm{b}_{\mathrm{i}-2, \mathrm{j}}=\mathrm{b}_{\mathrm{ij}}-\mathrm{b}_{\mathrm{i}-1, \mathrm{j}}-\mathrm{b}_{\mathrm{i}-2, \mathrm{j}}=\mathrm{q}_{\mathrm{ij}}$.

Dolayısıyla $\mathbf{A}_{\mathrm{n}} \mathbf{B}_{\mathrm{n}}=\mathbf{N}_{\mathrm{n}}$. Benzer şekilde $\mathbf{B}_{\mathrm{n}} \mathbf{A}_{\mathrm{n}}=\mathbf{R}_{\mathrm{n}}$ olduğu gösterilir.

$\mathbf{F}_{\mathrm{n}}$; Fibonacci matrisi, Tanım 2.3 ile verilen $\mathbf{A}_{\mathrm{n}}$ matrisinin tersine eşit olduğu için aşağıdaki teorem doğrudan sağlanır.

Teorem 3.7. $\boldsymbol{B}_{n}$; Bell matrisi olmak üzere

$$
\boldsymbol{B}_{n}=\boldsymbol{F}_{n} \boldsymbol{N}_{n}=\boldsymbol{R}_{n} \boldsymbol{F}_{n}
$$

şeklinde çarpanlarına ayrllabilir (Wang,2008). Bu faktorizasyonlar, $1 \leq k \leq n$ için $b_{n k}=\sum_{\ell=k}^{n} F_{n-\ell+1}\left(b_{\ell k}-b_{\ell-1, k}-b_{\ell-2, k}\right)=\sum_{\ell=k}^{n}\left(b_{n \ell}-b_{n, \ell+1}-b_{n, \ell+2}\right) F_{\ell-k+1}$. $E_{n}=(1,1, \cdots, 1)^{T}$ olmak üzere, $\boldsymbol{B}_{n} E_{n}=\boldsymbol{R}_{n} \boldsymbol{F}_{n} E_{n}$ ve Sonuç 3.1.1'den $F_{1}+F_{2}+\cdots+F_{n-2}=F_{n}-1$ olurve (16)'dan

$$
\sum_{k=1}^{n} b_{n k}=\sum_{k=1}^{n}\left(b_{n k}-b_{n, k+1}-b_{n, k+2}\right)\left(F_{k+2}-1\right)(\text { Wang,2008). }
$$

\section{Sonuç ve Öneriler}

$\mathrm{Bu}$ çalışmada Fibonacci, Pascal, Stirling ve Bell matrisleri ile ilgili bazı kombinasyonel özdeşlikler incelenmiştir. Bu özel yapıdaki matrislerin çeşitli normları ve şart sayıları hesaplanabilir, ayrıca bu matrislere benzer yapıda elemanları olan Lucas, Harmonik ve 
Catalan matrisleri tanımlanarak aralarındaki ilişkiler ve yeni kombinasyonel özdeşlikler incelenebilir.

\section{Kaynaklar}

Aigner M (1999). A characterization of the Bell numbers, Discrete Mathematics 205: 207210.

Ayber N (2003). Fibonacci sayıları, Matematik Dünyası Dergisi Kış: 56-57.

Cheon GS, Kim JS (2001). Stirling matrix via Pascal matrix, Linear Algebra and Its Applications 329: 49-59.

Çam Ş (2005). Stirling sayıları, Matematik Dünyası Dergisi Bahar: 30-34.

Edelman A, Strang G (1993). Pascal matrices, American Mathematical Monhtly 100: 372376.

Lee GY, Kim JS, Cho SH (2003). Some combinatorial identities via Fibonacci numbers, Discrete Applied Mathematics 13: 527-534.

Lee GY, Kim JS, Lee SG (2002). Factorizations and eigenvalues of Fibonacci and symmetric Fibonacci matrices, Fibonacci Quarterly 40(3): 203-211.

Rogers DG (1977). Pascal triangles, Catalan numbers and renewal arrays, Discrete Mathematics 22: 301-310.

Tang Z, Duraiswami R, Gumerov N (2004). Fast algorithms to compute matrix vector products for Pascal matrices, UMIACS-TR-08, CS-TR-4363.

Vajda S (1987). Fibonacci \& Lucas numbers and the golden section theory and applications, John Wiley \& Sons, London.

Wang W, Wang T (2008). Identities via Bell matrix and Fibonacci matrix, Discrete Applied Mathematics 156: 2793-2803.

\section{NOT: Bu çalışma “Bazı Özel Matrisler ve Kombinasyonel Özdeşlikler” başlıklı yükssek lisans tezinden üretilmiştir.}

